\title{
Low Anti-Mullerian Hormone Levels Are Associated with the Severity of Anxiety Experienced by Healthcare Professionals During the COVID-19 Pandemic
}

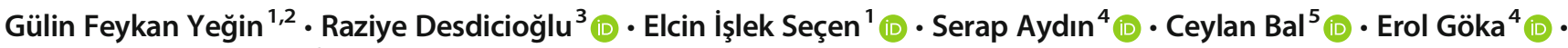 \\ Huseyin Levent Keskin ${ }^{6}$ (B)
}

Received: 28 March 2021 / Accepted: 27 May 2021 / Published online: 8 June 2021

(C) Society for Reproductive Investigation 2021

\begin{abstract}
The objective of this study is to investigate a possible correlation between anxiety status and anti-Mullerian hormone (AMH) levels among healthcare professionals who provide medical care directly to COVID-19-positive patients during the recent pandemic. Fifty-two healthcare professionals (nurses, midwives, and residents) who provide medical care directly to COVID19-positive patients in inpatient clinics or intensive care units were enrolled in this study. Serum AMH levels were analyzed to reflect ovarian reserve. The Beck Anxiety Inventory (BAI) and the State-Trait Anxiety Inventory (STAI-S and STAI-T, respectively) were completed by participants to assess their anxiety status. A linear regression model with participant age as the constant variable was applied to analyze the relationship between inventory scale scores and AMH levels. P-values less than 0.05 were considered statistically significant. The mean AMH value was significantly lower for the participants in the moderate/severe anxiety group compared to the minimal/mild anxiety group $(\mathrm{p}=0.007)$. A linear regression analysis revealed a significant negative correlation between AMH levels and both BAI $(B=-0.030$, standard error $=0.010, p=0.004)$ and STAI-S and STAI-T scores when age was controlled (both $p=0.003$ ). The severity of anxiety experienced during the recent COVID-19 pandemic among healthcare professionals, who provide medical care directly to COVID-19-positive patients, is found to be related to low AMH levels.
\end{abstract}

Keywords Anti-Mullerian hormone $\cdot$ Anxiety $\cdot$ COVID-19

Gülin Feykan Yeğin

gulin_yegin@hotmail.com

1 Department of Gynecology and Obstetrics, Ankara City Hospital, Ankara, Turkey

2 Ufuk Üniversitesi Cad., No:30/30 Çukurambar, Ankara, Turkey

3 Department of Gynecology and Obstetrics, Yıldırım Beyazıt University Faculty of Medicine, Ankara, Turkey

4 Department of Psychiatry, Ankara City Hospital, Ankara, Turkey

5 Department of Biochemistry, Yıldırım Beyazit University Faculty of Medicine, Ankara, Turkey

6 Department of Gynecology and Obstetrics, University of Health Sciences, Ankara, Turkey

\section{Introduction}

Acute global crises regarding health, such as the recent COVID-19 pandemic, greatly increase the work burden and risk of healthcare professionals compared with general society [1]. For example, the number of patients can increase rapidly, while the measures taken to protect healthcare providers and ensure continuity of resources may not be sufficiently responsive. As a consequence, the rate and severity of anxiety and depression symptoms among healthcare professionals who need to deal with multiple stressors increases. It has been reported that the rate of depression, insomnia, and anxiety among healthcare providers during the COVID-19 pandemic has reached $23.2 \%, 38.9 \%$, and $22.8 \%$, respectively [2]. Moreover, severe degrees of anxiety and depression symptoms have been reported to affect 2.2 to $14.5 \%$ of healthcare workers, during the COVID-19 pandemic [3].

Accumulating evidence suggests that experiencing psychiatric stress (mainly anxiety and depression) contributes to 
reproductive failure $[4,5]$. Multiple pathways have been proposed to mediate the relationship between stress and reproductive function, including activation of the hypothalamic pituitary adrenal axis which may result in a delay or inhibition of a luteinizing hormone surge and activation of the sympathetic system which can alter blood flow through the fallopian tubes and block gamete transport $[5,6]$.

Anti-Mullerian hormone (AMH) is secreted by granulosa cells of preantral and small antral follicles in the ovaries [7]. AMH is a member of the transforming growth factor- $\beta$ family of growth/differentiation factors and plays a role in ovarian follicle growth by inhibiting follicle stimulating hormonedependent oocyte recruitment [7]. Thus, AMH is a significant marker of oocyte quantity and quality and is considered a reliable biomarker of ovarian function [7].

Studies of the relationship between anxiety and ovarian reserve have reported inconsistent results [8-11]. For example, higher levels of psychological stress have been related to lower AMH levels, and this has strengthened the hypothesis that "psychological stress may affect ovarian reserve" $[8,9]$. In contrast, self-reported and current stresses were not associated with ovarian reserve in fertile and infertile women of reproductive age $[10,11]$.

Thus, to date, limited and contradictory evidence exists regarding the effect of psychological stress on serum AMH. Therefore, the aim of this study was to investigate a possible correlation between anxiety and AMH levels among healthcare professionals who provide medical care directly to COVID-19-positive patients during the current pandemic.

\section{Material and Methods}

A total of 95 healthcare professionals (including nurses, midwives, and residents) who provided medical care directly to COVID-19-positive patients at inpatient clinics or intensive care units between August and November 2020 were enrolled in this cohort study. This study was approved by our Institutional Review Board, and informed consent was obtained from all participants.

The subjects had no symptoms and received a negative result from a reverse transcriptase-polymerase chain reaction assay of a nasopharyngeal swab sample or computed tomography to detect severe acute respiratory syndrome coronavirus (SARS-CoV-2). The subjects also worked actively for at least 4 months providing COVID-19 services. The participants were homogenized according to the results of their initial evaluation in order to exclude factors that had been reported to likely affect AMH in previous studies [12]. In the initial evaluation, participants were administered an interview to establish their medical, surgical, family, and social histories. Healthcare workers with known/suspected pregnancy were not included. All healthcare providers with a history of systematic disorders, including polycystic ovary syndrome (PCOS), infertility, a life time history of depression/anxiety and related drug use, pelvic/abdominal surgery, malignancy, irregular menstrual periods, use of chemotherapy and/or radiotherapy, hormonal contraceptives, or any medications within 3 months prior to enrolling in this study were excluded. Women with polycystic ovary syndrome (PCOS) were excluded by using Rotterdam criteria. In addition, those who reported exposure to smoking, caffeine, alcohol, and/or recreational drug use, subjects older than 35 years, and subjects with a body mass index (BMI) $\geq 30 \mathrm{~kg} / \mathrm{m}^{2}$ were excluded due to the negative effects of these characteristics on ovarian reserve. After evaluating the initial 95 subjects, 37 subjects were excluded. Eleven subjects were excluded due to PCOS and an irregular menstruation period, hormonal drug use for these disorders, and thyroid dysfunction; five were excluded due to use of hormonal agents with the aim of contraception within 3 months; four were excluded due to use of antidepressant or anxiolytic agents; four were excluded for a BMI $\geq 30$ $\mathrm{kg} / \mathrm{m}^{2}$; two were excluded for being older than 35 years; three were excluded due to a history of endometrioma surgery; and eight were excluded because they did not accept the enrollment offered to them in this study. Therefore, a total of 58 subjects were enrolled in this study.

Venous blood samples were obtained from each of the participants during the early follicular phase of their menstrual period. Levels of AMH were analyzed with a human $\mathrm{AMH}$ enzyme-linked immunosorbent assay (Mini-VidasBioMerieux, Hazelwood, MO, USA).

The same day as the blood collection, participants completed the Beck Anxiety Inventory (BAI) and both the State-Trait Anxiety Inventory scales (STAI-S and STAI-T, respectively) to assess their anxiety status. All of the scales were scored by a single investigator (SA) who was blinded to the participants' ovarian reserve status. The BAI is a self-report questionnaire that evaluates the intensity of common somatic, affective, and cognitive anxiety symptoms, which have been experienced by the respondent within the previous 7 days. It consists of 21 items with Likert scale scoring. Definitive statements of anxiety symptoms are rated from 0 to 3 as follows: $0=$ not at all; 1 = mildly, but it didn't bother me much; 2 = moderately, it wasn't pleasant at times, and $3=$ severely, it bothered me a lot. The total score can range from 0 to 63 , and higher total scores indicate greater anxiety [13]. The total BAI scores are classified as a minimal anxiety level (score 0-7), a mild anxiety level (score 8-15), a moderate anxiety level (score 1625 ), and a severe anxiety level (score 26-63). In the current study, we combined the minimal and mild anxiety groups into a single group during analysis. The STAI consists of two subscales. STAI-S evaluates the feelings of an individual at a certain moment under a certain condition, while the STAI-T refers to anxiety as a personality trait regardless of circumstances [14]. The STAI includes 20 items, and participants 
are rated on a 4-point scale from 1 (almost never/not at all) to 4 (almost always/very much). The total score can range from 20 to 80 points, and a higher score indicates greater anxiety [14].

When AMH levels were analyzed, six participants had levels $\geq 6.0 \mathrm{ng} / \mathrm{mL}$. These cases involving excess AMH levels were excluded due to a possible relation with PCOS according to previous studies [12].

Laboratory results and anxiety scores for the 52 subjects in our cohort were compared to investigate a possible relationship between severity of anxiety and AMH level. Statistical analyses were performed by using SPSS (version 21.0; IBM Corporation, NY, USA). Data are expressed as the mean \pm standard deviation (SD) and median (interquartile range (IQR)) for all subjects and for each group. One-way analysis of variance (ANOVA) with the Bonferroni post hoc test was applied to compare the mean values between groups. Spearman correlation coefficient was used to analyze bivariate simple correlations between subject age and variables. A linear regression model with age controlled as the constant variable was applied to analyze a possible correlation between inventory scale scores and AMH levels. A correlation was indicated according to coefficients value (B), standard error (SE), and p-value. P-values less than 0.05 were considered statistically significant.

\section{Results}

The mean age of our cohort was $29.7 \pm 4.1$ years. The 52 participants examined were as follows: nulligravid $(n=22)$, nulliparous $(n=9)$, primiparous $(n=18)$, and multiparous $(n=$ 3 ). The mean AMH level for the cohort was $1.90 \pm 1.15 \mathrm{ng} /$ $\mathrm{mL}$. A more detailed AMH profile is presented in Table 1.

Based on the BAI scores obtained, there were 10 (19.2\%) subjects in the minimal anxiety group, $12(23.1 \%)$ subjects in the mild anxiety group, $14(26.9 \%)$ subjects in the moderate anxiety group, and $16(30.8 \%)$ subjects in the severe anxiety group. The mean AMH value for the minimal and mild anxiety groups was significantly higher than the mean AMH values for the other three groups $(F=5.574, p=0.007)$. In contrast, the mean AMH values were similar between the moderate and severe anxiety groups $(1.46 \pm 1.00 \mathrm{ng} / \mathrm{mL}$ vs. $1.50 \pm 0.89 \mathrm{ng} / \mathrm{mL}$, respectively) (Fig. 1).
When AMH levels were analyzed according to participant age, a significant negative correlation was observed $(\mathrm{r}=$ $-0.429, p=0.002$ ). However, the anxiety scores of the inventories were not significantly affected by subject age (BAI scores: $r=0.110, p=0.436$; STAI-S scores: $r=0.212, p=$ 0.132; STAI-T scores: $\mathrm{r}=0.132, \mathrm{p}=0.350$ ).

Linear regression analysis also revealed a significant negative correlation between AMH levels and both BAI (B = $-0.030, \mathrm{SE}=0.010, \mathrm{p}=0.004)$ and STAI-S and STAI-T scale scores when age was controlled $(\mathrm{B}=-0.024, \mathrm{SE}=0.008, \mathrm{p}=$ 0.003 and $\mathrm{B}=-0.029, \mathrm{SE}=0.009, \mathrm{p}=0.003$, respectively) (Figs. 2, 3, and 4).

\section{Discussion}

The principal implication of the current results is that a significant negative correlation was detected between AMH levels and BAI and STAI-S and STAI-T scale scores. These results add to the growing body of research, which supports the hypothesis that a negative correlation exists between anxiety and AMH levels.

Since late December 2019, healthcare providers have had extended working hours while dealing with an unexpected pandemic and having insufficient knowledge of the disease involved. Continuous use of personal protection equipment has further added to the physical exhaustion and mental pressure experienced by healthcare professionals [15]. Research performed during previous epidemics/pandemics has shown that healthcare providers encounter a broad range of psychological morbidities. In particular, concerns regarding fear, anxiety, and stigmatization were reported [1, 16]. The relationship between psychiatric traumas and suicide has also been documented [16, 17]. Fears over risk to health and social isolation can lead to psychological stress. Moreover, lack of personal protective equipment that can arise during a pandemic represents a stressor [1]. Studies of the MERS-CoV outbreak in 2014, which resulted in an excessive amount of anxiety among people in infested countries, revealed that the healthcare professionals involved were suffering extreme levels of stress, as this group of individuals was potentially facing the highest levels of infection risk [18, 19]. Furthermore, it was demonstrated that $30 \%$ of the infection
Table 1 AMH profile of our cohort $(\mathrm{n}=52)$

\begin{tabular}{lccc}
\hline Characteristic & Mean \pm SD & Median (IQR) & Min.-Max. \\
\hline Age $(\mathrm{y})$ & $29.7 \pm 4.1$ & $29.0(6.75)$ & $20.0-35.0$ \\
AMH $(\mathrm{ng} / \mathrm{mL})$, overall & $1.90 \pm 1.15$ & $1.68(1.77)$ & $0.01-5.01$ \\
Minimal/mild anxiety $(\mathrm{n}=22)$ & $2.47 \pm 1.20$ & $2.60(1.53)$ & $0.21-5.01$ \\
Moderate anxiety $(\mathrm{n}=14)$ & $1.46 \pm 1.00$ & $1.36(1.19)$ & $0.01-3.30$ \\
Severe anxiety $(\mathrm{n}=16)$ & $1.50 \pm 0.89$ & $1.34(0.93)$ & $0.18-3.59$ \\
\hline
\end{tabular}

$A M H$ anti-Mullerian hormone, $I Q R$ interquartile range, $m L$ milliliter, $n g$ nanogram, $S D$ standard deviation, $y$ years 


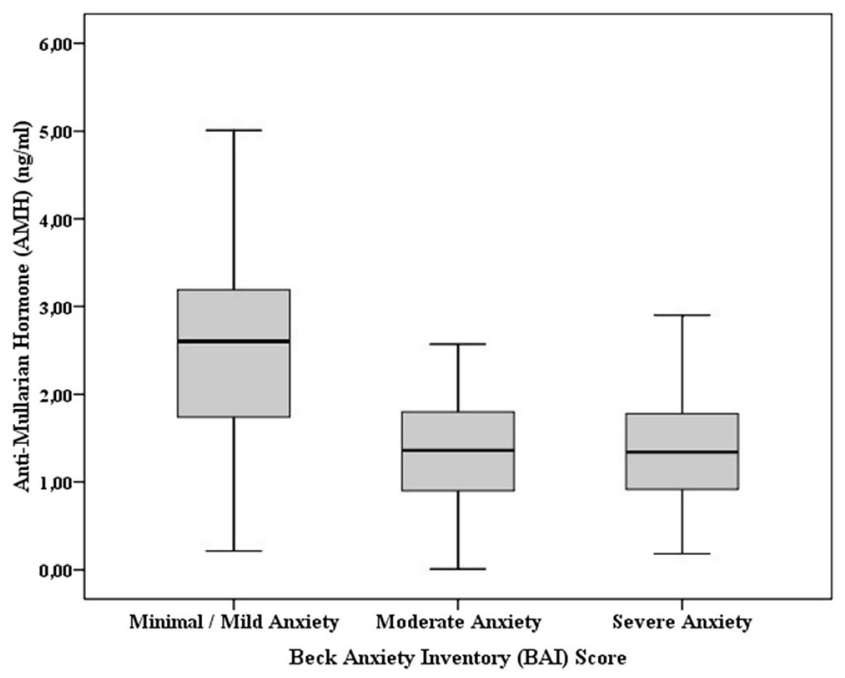

Fig. 1 Boxplot of mean AMH levels according to anxiety intensity determined by BAI scores

cases involved medical staff, with most of the infection spread occurring within the boundaries of hospitals [18, 19]. More recently, in a study conducted among frontline physician trainees during the COVID-19 pandemic who were exposed to COVID-19-positive subjects, they exhibited a higher prevalence of stress and were more likely to suffer from burnout compared to a non-exposed group of trainees [20]. Additionally, it was observed that the female trainees were more affected by stress [20].

The relationship between psychological stress and reproductive functions has not been fully elucidated. Biological responses to stress may lead to increased levels of stress hormones, which lessens granulosa cell function and affects follicular maturation. This in turn can affect the final number of oocytes [21]. Psychological stress can also have a positive association with antral follicular count in younger women [22]. However, an age-related reduction in antral follicular

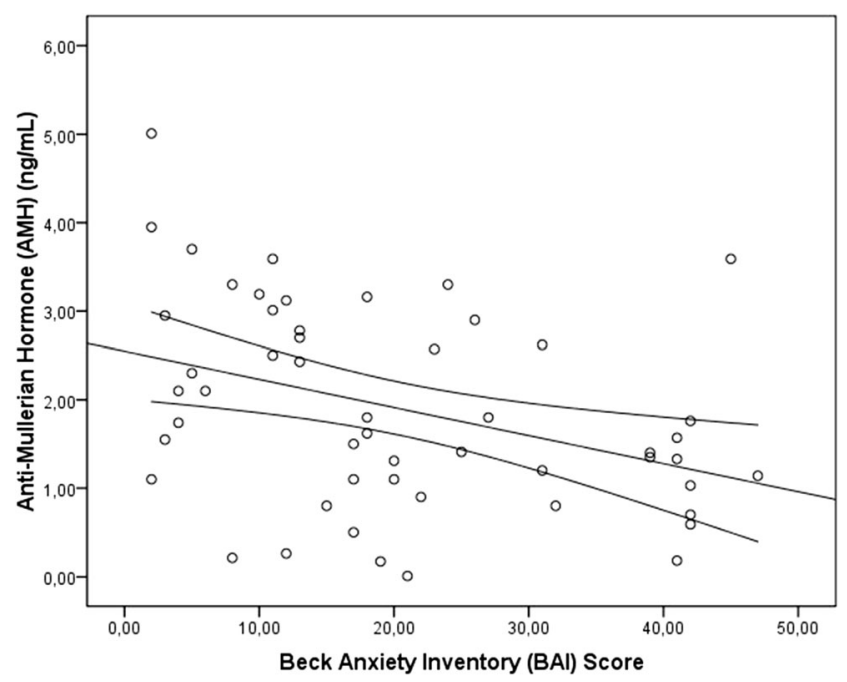

Fig. 2 Linear regression analysis of BAI scores and AMH levels

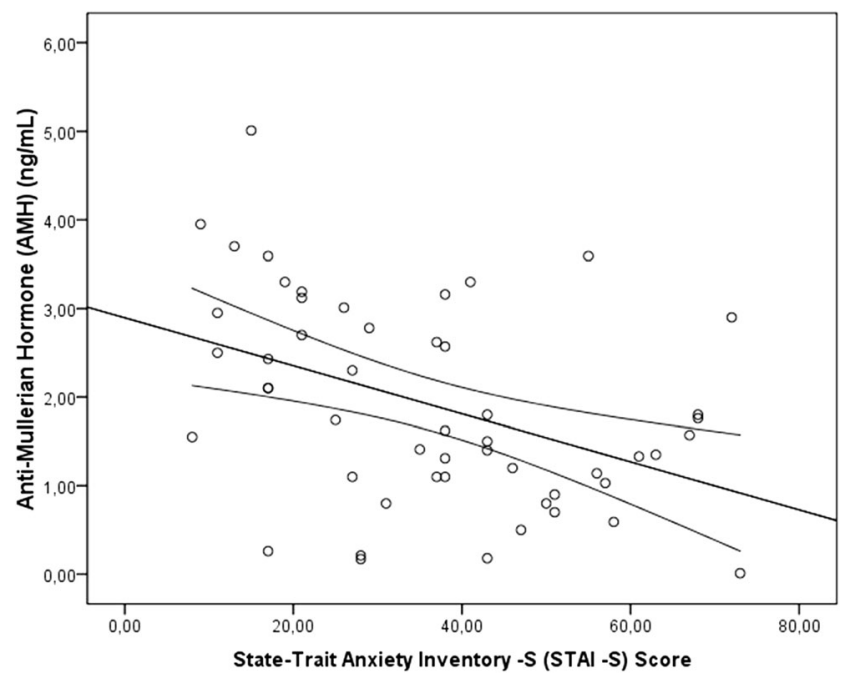

Fig. 3 Linear regression analysis of STAI-S scores and AMH levels

count can be precipitated by elevated stress levels in all age groups [22]. Furthermore, a depressive clinical condition can moderate the effects of stress on this decline [23].

Previously, a cross-sectional study conducted among Chinese women with unexplained infertility demonstrated a negative correlation between salivary alpha-amylase, a biomarker of psychological stress, and serum AMH [8]. In contrast, a separate cross-sectional study found no relationship between stress and serum AMH in infertile women with unexplained infertility [10]. More recently, a case-control study of 190 women found that self-reported stress and psychological distress were not associated with serum AMH levels in either infertile or fertile women of reproductive age [11]. Meanwhile, a cross-sectional study of 89 infertile women identified "chronic" lifetime psychosocial stressors such as a personal history of abuse or recreational drug use as endamaging factors, rather than "current" stress, based on morning serum cortisol level and dysphoric mood score data [24].

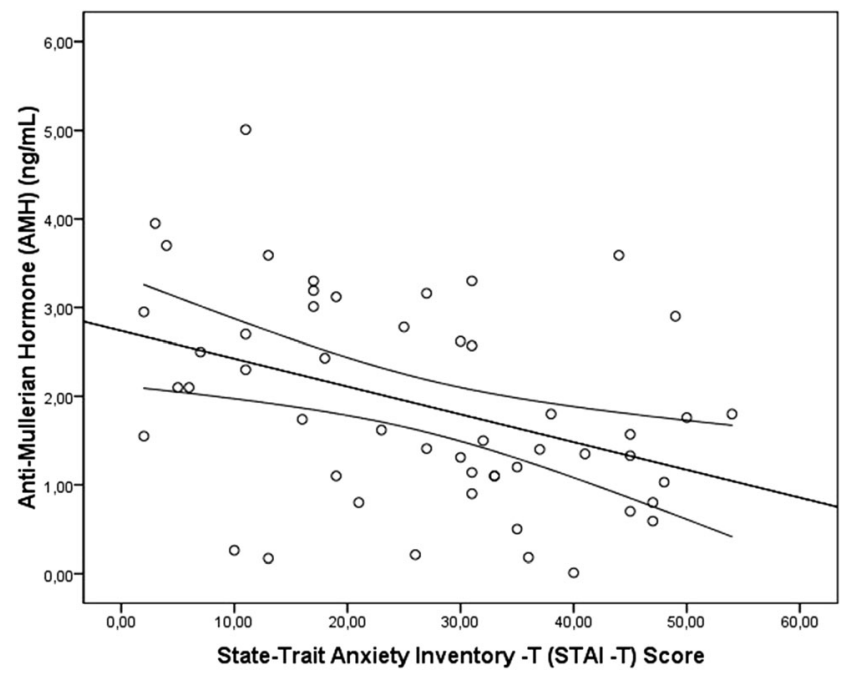

Fig. 4 Linear regression analysis of STAI-T scores and AMH levels 
The main strength of the present study is its objectivity, which is achieved with the use of multiple scales to assess severity of anxiety. In addition, the BAI and STAI scales have been shown to be reliable and valid instruments in measuring anxiety [25]. For example, when the performance of various self-report measures of anxiety was examined, the BAI exhibited the highest correlations with severity of anxiety, favorable discriminant validity, and a fair balance between specificity and sensitivity with a cutoff point of 15/16 [25]. Furthermore, both the STAI scales have demonstrated good discriminant validity, with the "Trait" scale exhibiting greater sensitivity and specificity for a diagnosis of anxiety compared to other scales [25]. Another strength of the present study was its elimination of factors, which have been reported to likely affect AMH in previous studies (see "Material and Methods" section). In contrast, the smaller sample size and broadening of exclusion criteria represent potential limitations of the present study. Current study is also limited by the lack of AMH levels prior to this investigation, which would directly test if the resulting anxiety values and AMH levels are a result of the COVID-19 pandemic. Furthermore, a lack of information regarding AMH levels in healthcare professionals during other pandemics prevented a comparison of our data with other pandemics. An additional uncontrolled factor is the lack of stress levels prior to current investigation, which would provide opportunity for comparison with current data.

The results of the current study suggest that low AMH levels among healthcare professionals during the recent COVID-19 pandemic are related to anxiety severity. Significantly lower AMH levels were found among the individuals who received higher inventory scores. The insights gained from these data may help elucidate the relationship between anxiety and reproductive functions. The present findings also have important implications for future studies by demonstrating that the psychological stress induced by a virus/infection can lead to secondary consequences. Further research including comparison of AMH levels after widespread vaccination would be beneficial to support current findings.

Data Availability The data that support the findings of the study are available from the corresponding author upon reasonable request.

\section{Declarations}

Ethics Approval The study was approved by the Institutional Review Board (No:26379996/48).

Consents to Participate and for Publication Informed consent was obtained from all individual participants included in the study.

Conflict of Interest The authors declare no competing interests.

\section{References}

1. Galbraith N, Boyda D, McFeeters D, Hassan T. The mental health of doctors during the COVID-19 pandemic. BJPsych Bull. 2021;45(2):93-7.

2. Pappa S, Ntella V, Giannakas T, Giannakoulis VG, Papoutsi E, Katsaounou P. Prevalence of depression, anxiety and insomnia among healthcare workers during the COVID-19 pandemic: a systematic review and meta-analysis. Brain Behav Immun. 2020;88: 901-7.

3. Bohlken J, Schömig F, Lemke MR, Pumberger M. Riedel- Heller SG. COVID-19 pandemic: stress experience of healthcare workers - a short current review. Psychiatr Prax. 2020;47(4):190-7.

4. Rooney KL, Domar AD. The relationship between stress and infertility. Dialogues Clin Neurosci. 2018;20(1):41-7.

5. Louis GM, Lum KJ, Sundaram R, Chen Z, Kim S, Lynch CD, et al. Stress reduces conception probabilities across the fertile window: evidence in support of relaxation. Fertil Steril. 2011;95(7):2184-9.

6. Ferin M. Clinical review 105: Stress and the reproductive cycle. J Clin Endocrinol Metab. 1999;84(6):1768-74.

7. Iwase A, Nakamura T, Osuka S, Takikawa S, Goto M, Kikkawa F. Anti-Müllerian hormone as a marker of ovarian reserve: what have we learned, and what should we know? Reprod Med Biol. 2015;15(3):127-36.

8. Dong YZ, Zhou FJ, Sun YP. Psychological stress is related to a decrease of serum anti-Müllerian hormone level in infertile women. Reprod Biol Endocrinol. 2017;15:51.

9. Nakamura K, Sheps S, Arck PC. Stress and reproductive failure: past notions, present insights and future directions. J Assist Reprod Genet. 2008;25:47-62.

10. Vitek W, Barrett ES, Hoeger K, Diamond MP, Cedars M, Steiner AZ. Psychological stress and ovarian reserve among women with unexplained infertility. Fertil Steril. 2016;106(3):e66.

11. Lawal OI, Akinyemi JO, Yusuff JO, Okunlola MA. Perceived stress, psychological distress and serum anti-Müllerian hormone levels among infertile and fertile women in North-central Nigeria. Middle East Fertil Soc J. 2020;25:19.

12. Moolhuijsen LME, Visser JA. Anti-Müllerian hormone and ovarian reserve: update on assessing ovarian function. J Clin Endocrinol Metab. 2020;105(11):3361-73.

13. Beck AT, Steer RA. Manual for the Beck Anxiety Inventory. San Antonio, TX: Psychological Corporation; 1993.

14. Spielberger CD, Gorsuch RL, Lushene R, Vagg PR, Jacobs L. Manual for the State-Trait. Anxiety Inventory Palo Alto, vol. 22. Calif Consulting Psychologists Press. 1970;2020:1-24.

15. Talaee N, Varahram M, Jamaati H, Salimi A, Attarchi M, Kazempour Dizaji M, et al. Stress and burnout in health care workers during COVID-19 pandemic: validation of a questionnaire. Z Gesundh Wiss. 2020;6:1-6.

16. McFeeters D, Boyda D, Siobhan O. Patterns of stressful life events: distinguishing suicide ideators from suicide attempters. Journal of affective disorders. 2015;175:192-8.

17. Stanley IH, Hom MA, Joiner TE. A systematic review of suicidal thoughts and behaviors among police officers, firefighters, EMTs, and paramedics. Clinical Psychology Review. 2016;44:25-44.

18. Memish Z, et al. Screening for Middle East respiratory syndrome coronavirus infection in hospital patients and their healthcare worker and family contacts: a prospective descriptive study. Clin Microbiol Infect. 2014;20:469-74.

19. Oboho IK, Tomczyk SM, al-Asmari AM, Banjar AA, al-Mugti H, Aloraini MS, et al. 2014 MERS-CoV outbreak in Jeddah-a link to health care facilities. N Engl J Med. 2015;372:846-54. 
20. Kannampallil TG, Goss CW, Evanoff BA, Strickland JR, McAlister RP, Duncan J. Exposure to COVID-19 patients increases physician trainee stress and burnout. PLoS ONE. 2020;15(8):e0237301.

21. Ebbesen SMS, Zachariae R, Mehlsen MY, Thomsen D, Højgaard A, Ottosen L, et al. Stressful life events are associated with a poor in-vitro fertilization outcome: a prospective study. Hum Reprod. 2018;24(9):2173-82.

22. Bleil ME, Adler NE, Pasch LA, Sternfeld B, Gregorich SE, Rosen MP, et al. Psychological stress and reproductive aging among premenopausal women. Hum Reprod. 2012;27(9):2720-8.

23. Bleil ME, Adler NE, Pasch LA, Sternfeld B, Gregorich SE, Rosen MP, et al. Depressive symptomatology, psychological stress, and ovarian reserve: a role for psychological factors in ovarian aging? Menopause. 2013;19(11):1176-85.

24. Pal L, Bevilacqua K, Santoro NF. Chronic psychosocial stressors are detrimental to ovarian reserve: a study of infertile women. J Psychosom Obstet Gynaecol. 2010;31:130-9.

25. Dennis RE, Boddington SJA, Funnell NJ. Self-report measures of anxiety: are they suitable for older adults? Aging \& Mental Health. 2007;11:668-77.

Publisher's Note Springer Nature remains neutral with regard to jurisdictional claims in published maps and institutional affiliations. 